\title{
Neuralgic amyotrophy associated with COVID-19 infection: a case report and review of the literature
}

\author{
Ismail Ibrahim Ismail ${ }^{1}$ (D) Ehab A. Abdelnabi ${ }^{2} \cdot$ Jasem Y. Al-Hashel ${ }^{1,3} \cdot$ Raed Alroughani $^{4} \cdot$ Samar Farouk Ahmed $^{1,5}$
}

Received: 31 January 2021 / Accepted: 16 March 2021 / Published online: 20 March 2021

(C) Fondazione Società Italiana di Neurologia 2021

\section{Introduction}

Since the spread of severe acute respiratory syndrome coronavirus 2 (SARS-CoV-2) around the world, and the declaration of coronavirus disease 2019 (COVID-19) pandemic, it has become increasingly evident that several neurological manifestations are associated with this disease, involving both central and peripheral nervous systems [1].

Neuralgic amyotrophy is an uncommon clinical syndrome characterized by initial severe neuropathic pain and patchy weakness of the shoulder and arm muscles, usually involving the upper and middle trunks of the brachial plexus, followed by slow recovery. Neuralgic amyotrophy can be idiopathic or hereditary and is usually unilateral in $70 \%$ of cases [2].

Etiology and pathophysiology of neuralgic amyotrophy are still unclear; several precipitating factors had been reported including post-infectious, post-vaccination, post-operative, and post-traumatic, [3] with recent viral illness being the most common associated trigger [4].

To our knowledge, only 3 case reports have documented neuralgic amyotrophy in association with SARS-CoV-2 infection in the literature [5-7]. Herein, we present a case of severe, bilateral asymmetric neuralgic amyotrophy in a 32-year-old male, in association with laboratory-confirmed infection with

SARS-CoV-2. This represents the first case from the Middle East and North Africa (MENA) region, to the best of our knowledge, expanding the spectrum of neurological involvement in association with COVID-19 infection.

\section{Case report}

A 32-year-old right-handed male with no relevant past medical history developed fever, cough, and generalized body pain in November 2020. He tested positive for SARS-CoV-2 via a nasopharyngeal swab reverse transcription-polymerase chain reaction (RT-PCR). He was hospitalized in a specialized institution for COVID19 and received oxygen, antibiotics, vitamins, and anticoagulants and was discharged after 7 days. During hospital stay, he experienced severe pain at the left shoulder, aggravated by touch and movement, for which he received acetaminophen with minimal relief. The pain involved the right shoulder one week later.

After discharge, the pain intensified and progressed to involve both forearms and hands. Two weeks from the onset, he started to develop progressive proximal weakness of both upper limbs, more on the left side, in addition to developing bilateral hand numbness, and an area of

Ismail Ibrahim Ismail

dr.ismail.ibrahim2012@gmail.com

Ehab A. Abdelnabi

afandena@gmail.com

Jasem Y. Al-Hashel

jasemkumsa@hotmail.com

Raed Alroughani

alroughani@gmail.com

Samar Farouk Ahmed

samerelshayb@hotmail.com
Department of Neurology, Ibn Sina Hospital, BOX 25427, Gamal Abdel Nasser Street, Sabah Medical Area, 13115 Kuwait, Kuwait

2 Department of Radiology, Ibn Sina Hospital, Kuwait, Kuwait

3 Health Sciences Centre, Department of Medicine, Kuwait University, Kuwait, Kuwait

4 Division of Neurology, Department of Medicine, Amiri Hospital, Sharq, Kuwait, Kuwait

5 Department of Neurology and Psychiatry, Minia University, Minya, Egypt 
sensory loss over his left shoulder and radial forearm. He was initially evaluated by general physician and orthopedic surgeon and received pregabalin $300 \mathrm{mg} /$ day, tramadol hydrochloride $50 \mathrm{mg}$ twice daily, and local injection of steroids and lidocaine in both shoulders. He showed little improvement and was referred to our neurology outpatient clinic after 5 weeks of onset. He reported having severe pain ranging from 7 to 10 out of 10 on numerical rating scale (NRS), where 0 is no pain and 10 is the most severe pain.

Findings of his systemic examination were normal. On neurological examination, he was alert, conscious, and oriented with normal speech and higher mental functions. Cranial nerve assessment was normal. Motor examination on the left side showed winging of scapula, weakness of shoulder abduction and flexion of Medical Research Council (MRC) grade 1/5, weakness of shoulder external rotation and elbow flexion of MRC grade $2 / 5$, and weakness of flexion of the distal interphalangeal joints of the thumb and index finger "OK sign" of MRC grade 4/5. On the right side, he had weakness of shoulder abduction, shoulder flexion, and elbow flexion of MRC grade 4/5. Minimal atrophy was observed on both shoulders, more on the left side. Motor examination of both lower limbs was normal. Deep tendon reflexes were absent all over the body, except for right triceps and brachioradialis reflexes. Sensory assessment showed reduced sensation over the right and left shoulders, and left forearm. Planter response was downgoing bilaterally.

Electrophysiological studies (nerve conduction tests and electromyography) were performed 5 weeks after initial presentation. It revealed sensory and motor axonal involvement, in the form of low amplitude of compound motor action potentials of musculocutaneous, axillary, and suprascapular nerves on both sides, and long thoracic and anterior interosseous nerve on the left side, with patchy denervation. The picture was compatible with bilateral neuralgic amyotrophy. A magnetic resonance imaging (MRI) showed hyperintense T2-weighted signal of supra- and infraspinatus muscles, indicating intramuscular edema, with normal cervical spine and both brachial plexuses, further confirming the diagnosis (Fig. 1).

An extensive blood workup showed normal complete blood count; renal and liver functions; serum electrolytes; creatine kinase; inflammatory markers (erythrocyte sedimentation rate, C-reactive protein); serum vitamins B1, B6, B12, and folate; protein electrophoresis; immunoglobulin essay; thyroid function; and antithyroid autoantibodies. There was no serological evidence of infection with hepatitis B, C, and E; herpes simplex virus (HSV);
Fig. 1 Magnetic resonance imaging (MRI) of cervical spine and both brachial plexuses in a 32-year-old male. a Coronal 3D T2-weighted images with fat suppression using sampling perfection with applicationoptimized contrasts by using flip angle evolution (SPACE) sequence, showing abnormal signal that involved the rotator cuff muscles bilaterally (arrows); b coronal 3D T2-weighted image through the brachial plexus showing no pathological changes; and $\mathbf{c}$ sagittal T2-weighted image of the cervical spine showing no myelopathy or compressing masses

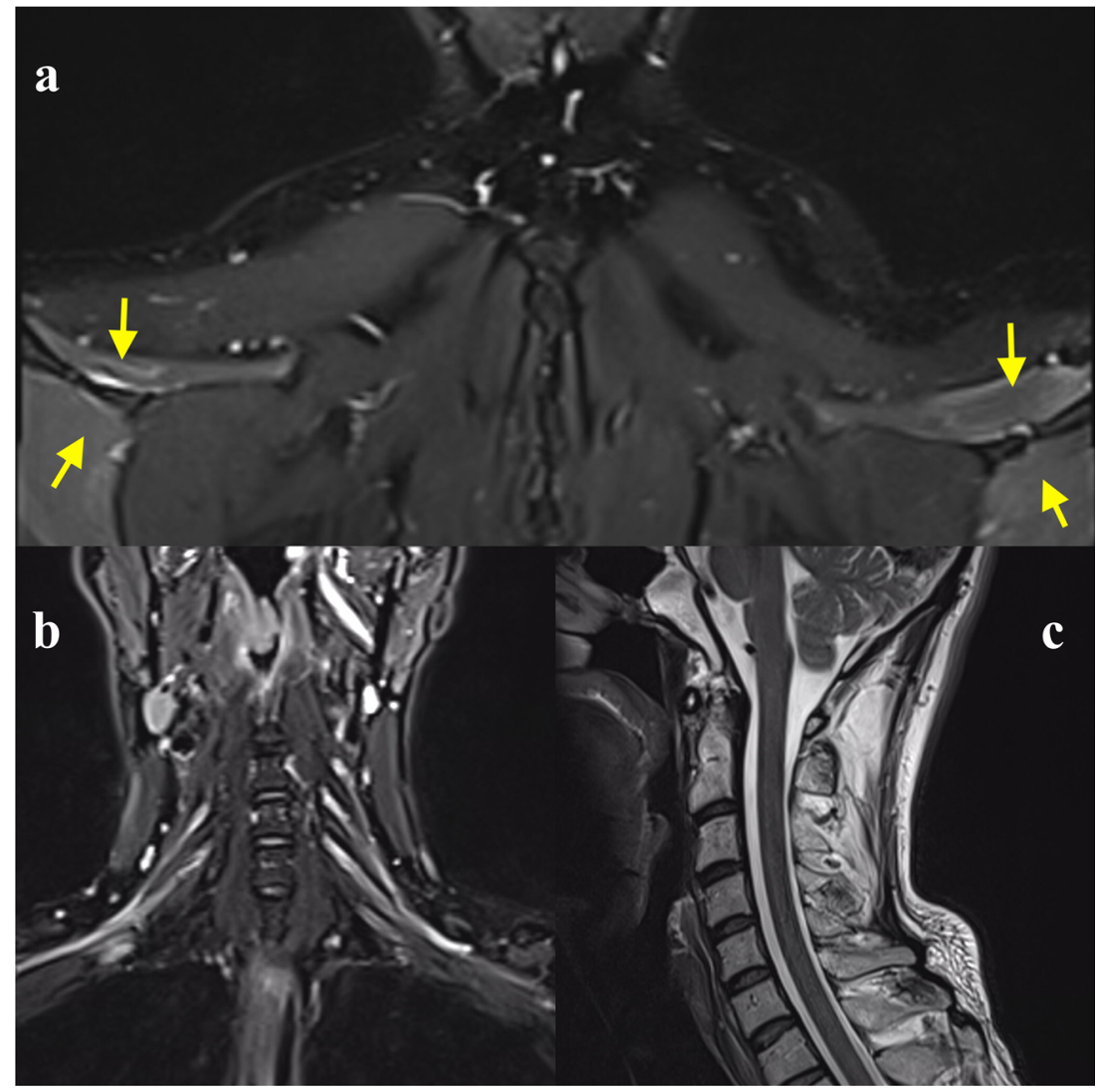


human immunodeficiency virus (HIV); Lyme; syphilis; or toxoplasma. A panel for vasculitis and autoimmune antibodies yielded negative results. Cerebrospinal fluid (CSF) analysis showed normal protein and glucose levels, with no WBCS, negative culture and sensitivity, and gram stain for bacterial infection. Polymerase chain reaction screening for neurotropic viruses was negative in serum and CSF.

The patient was commenced on intravenous methylprednisolone $1000 \mathrm{mg}$ per day for 5 days, but it was stopped due to dermatological side effects. He received a course of intravenous immunoglobulins (IVIg) in a dose of $25 \mathrm{~g} /$ day for 5 days. There was partial relief of pain with no improvement in muscle power. His motor and sensory examinations remained the same after 8 weeks of symptoms onset.

\section{Discussion}

Involvement of peripheral nervous system in patients with COVID-19 is on the rise. In an early study from China [8], it was estimated to affect $8.9 \%$ of patients; however, it is more likely underreported, requiring further electrophysiological studies to determine its true incidence and characteristics. This involvement may result from direct neuro-invasion or from an autoimmune, post-infectious mechanism [8]. Understanding the sequence and mechanism of events leading to neural damage in patients with COVID-19 might help in early diagnosis and treatment and highlights the need for prospective research on patterns of peripheral and central nervous system involvement in patients with COVID-19 [9].

Neuralgic amyotrophy is a rare form of peripheral neuropathy that has been rarely reported in association with COVID-19, to our knowledge. A PubMed search was performed using the following combinations of keywords: neuralgic amyotrophy, SARS-CoV-2, COVID-19, and brachial plexopathy. It revealed only 3 case reports in the literature [5-7], till the time of writing (Table 1). The report of Cacciavillani et al. includes a patient who developed pure sensory neuralgic amyotrophy, possibly related to infection with SARS-CoV-2, without any clinical or electrophysiological signs of motor nerve involvement. The report of Siepmann et al. reported a patient with sole median nerve involvement, as a rare clinical manifestation of neuralgic amyotrophy. A third report by Mitry et al. showed hyperintense T2-weighted signal of the supraspinatus, infraspinatus, teres minor, teres major, and trapezius muscles on MRI, with initial improvement on oral steroids. These different presentations of postCOVID-19 neuralgic amyotrophy highlight the possible variability of symptoms in patients with peripheral nervous system involvement with SARS-CoV-2 infection, and the importance of detailed assessment of patients with COVID-19 for neurological deficits [9].

Neuralgic amyotrophy is a distinct disorder that is more common in males, predominantly affecting the roots of the brachial plexus, and characterized by excruciating neuropathic pain followed by multifocal paresis and sensory loss in C5, C6, and less frequently in $\mathrm{C} 7$ distribution. It has two subtypes, hereditary neuralgic amyotrophy (HNA) and idiopathic neuralgic amyotrophy (INA), and is estimated to affect 2-3 cases/ 100,000 inhabitants/year. The involvement is mainly unilateral in $70 \%$ of cases, and bilateral or asymmetric in the other $30 \%$. Diagnosis is confirmed by electrophysiological studies and sometimes with evidence of strain in the supra and infraspinatus muscles on MRI $[10,11]$.

The etiology of the disease is not well understood; however, an immune-mediated reaction, that is triggered by earlier or concomitant infection is believed to be the cause in $43 \%$ of cases [12]. Some features in our report are different from the other published cases. First, the bilaterality of pain and weakness, which is an uncommon feature of neuralgic amyotrophy in itself. Second, the very early onset of symptoms during the initial presentation of COVID19 , suggesting a probable direct viral neuroinvasion, rather than an autoimmune post-infectious response against SARS-CoV-2 [13]. Moreover, our report showed hyperintense T2-weighted signal of supra and infraspinatus muscles in MRI. This is explained by denervation of skeletal muscles in the distribution of the brachial plexus nerves, due to intramuscular edema [14]. MRI helpsin ruling out other etiologies of painful shoulder weakness.

Treatment of neuralgic amyotrophy has not been validated, in the absence of randomized controlled trials (RCTs). A reasonable protocol for adults was found to be oral prednisone (1 $\mathrm{mg} / \mathrm{kg}$ per day) for 1 week, followed by tapering of $10 \mathrm{mg}$ per day during the second week [15]. Intravenous immunoglobulin (IVIg) has also been proposed as a treatment [16], in case of contraindication to steroids or the emergence of side effects, similar to our case.

\section{Conclusion}

Our case adds to the growing body of evidence of peripheralnervous system involvement in COVID-19 patients. Despite being a rare disease, neuralgic amyotrophy should be suspected and ruled out in COVID-19 patients, presenting with severe pain and weakness of the shoulder and arm muscles. This study highlights the importance of detailed assessment of patients with COVID-19 with neurological deficits, to avoid delay in diagnosis and allow for early management. 


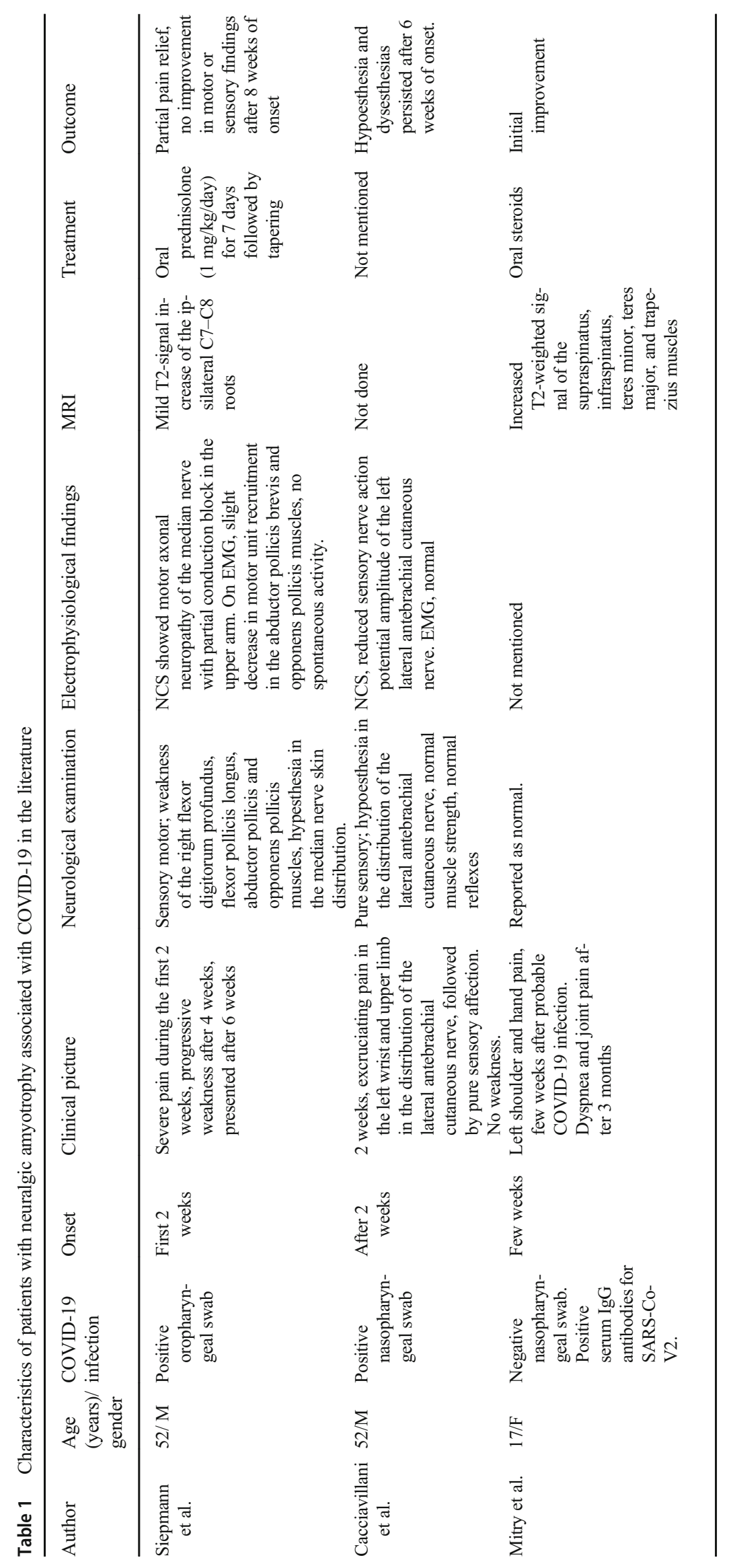


Author contribution III, EAA, and SFA were involved with acquisition of data. III, RA, JYA, and SFA treated the patient. III, and SFA wrote the initial manuscript. All authors critically appraised and revised it. All authors read and approved the final manuscript.

\section{Declarations}

Conflict of interest The authors declare no competing interests.

Ethical approval This case report was approved by the Institutional Ethics Committee.

Informed consent Written informed consent for publication of this case report was obtained from the patient.

\section{References}

1. Pezzini A, Padovani A (2020) Lifting the mask on neurological manifestations of COVID-19. Nat Rev Neurol 16:636-644. https://doi.org/10.1038/s41582-020-0398-3

2. van Alfen N (2011) Clinical and pathophysiological concepts of neuralgic amyotrophy. Nat Rev Neurol 7:315-322. https://doi.org/ 10.1038/nrneurol.2011.62

3. Feinberg JH, Radecki J (2010) Parsonage turner syndrome. HSS J 6(2):199-205

4. Fibuch EE, Mertz J, Geller B (1996) Postoperative onset of idiopathic brachial neuritis. Anesthesiology. 84(2):455-458

5. Siepmann T, Kitzler HH, Lueck C (2020) Neuralgic amyotrophy following infection with SARS-CoV-2. Muscle Nerve 62(4):E68E70. https://doi.org/10.1002/mus.27035

6. Cacciavillani M, Salvalaggio A, Briani C (2020) Pure sensory neuralgic amyotrophy in COVID-19 infection. Muscle Nerve

7. Mitry MA, Collins LK, Kazam JJ, Kaicker S, Kovanlikaya A (2020) Parsonage-turner syndrome associated with SARS-CoV2 (COVID-19) infection. Clin Imaging 72:8-10
8. Mao L, Jin H, Wang M, Hu Y, Chen S, He Q, Chang J, Hong C, Zhou Y, Wang D, Miao X, Li Y, Hu B (2020) Neurologic manifestations of hospitalized patients with coronavirus disease 2019 in Wuhan, China. JAMA Neurol 77:e201127. https://doi.org/10.1001/ jamaneurol.2020.1127

9. Siepmann T, Kitzler HH, Reichmann H, Barlinn K (2021) Variability of symptoms in neuralgic amyotrophy following infection with SARS-CoV-2. Muscle Nerve 63(1):E8-E9. https://doi. org/10.1002/mus.27084

10. Van Eijk JJ, Groothuis JT, Van Alfen N (2016) Neuralgic amyotrophy: an update on diagnosis, pathophysiology, and treatment. Muscle Nerve 53(3):337-350

11. Gstoettner C, Mayer JA, Rassam S, Hruby LA, Salminger S, Sturma A, Aman M, Harhaus L, Platzgummer H, Aszmann OC (2020) Neuralgic amyotrophy: a paradigm shift in diagnosis and treatment. J Neurol Neurosurg Psychiatry

12. van Alfen N, Van Engelen BG (2006) The clinical spectrum of neuralgic amyotrophy in 246 cases. Brain. 129(2):438-450

13. Li YC, Bai WZ, Hashikawa T (2020) The neuroinvasive potential of SARS-CoV2 may play a role in the respiratory failure of COVID-19 patients. J Med Virol 92(6):552-555. https://doi.org/ $10.1002 /$ jmv. 25728

14. Scalf RE, Wenger DE, Frick MA, Mandrekar JN, Adkins MC (2007) MRI findings of 26 patients with parsonage-turner syndrome. Am J Roentgenol 189(1):W39-W44

15. van Alfen N, van Engelen BG, Hughes RA (2009) Treatment for idiopathic and hereditary neuralgic amyotrophy (brachial neuritis). Cochrane Database Syst Rev 3

16. Naito KS, Fukushima K, Suzuki S, Kuwahara M, Morita H, Kusunoki S, Ikeda SI (2012) Intravenous immunoglobulin (IVIg) with methylprednisolone pulse therapy for motor impairment of neuralgic amyotrophy: Clinical observations in 10 cases. Intern Med 51:1493-1500

Publisher's note Springer Nature remains neutral with regard to jurisdictional claims in published maps and institutional affiliations. 